\title{
Outcome and survival in systemic lupus erythematosus
}

Most rheumatologists are aware of the increased survival being reported in patients with systemic lupus erythematosus (SLE). Thus in 1954 the survival at Johns Hopkins University was less than $50 \%$ after four years. ${ }^{1}$ Throughout the following decades a progressive improvement in survival has been reported. Since 1980 four large studies, which included a total of 2699 patients, have reported remarkably consistent five year survival rates of $86-88 \%$ and 10 year survival rates of $76-79 \% .^{2-5} \mathrm{~A}$ number of factors probably contribute to the apparent increase in survival, including recognition of mild disease manifestations, improved diagnostic techniques, and earlier therapeutic intervention. Moreover, improvements in therapeutic modalities, such as more effective immunosuppressive agents, antibiotics, antihypertensive drugs, haemodialysis, and transplantation, may also have contributed.

As well as diagnostic and therapeutic factors, survival in SLE may be affected by several other influences, including the sex of the patients, age of onset, race, socioeconomic status, time between onset of disease and diagnosis, and distribution of organ system involvement. Published studies have produced some contradictory findings (reviewed by Ginzler and Schorn. ${ }^{6}$ ) For example, different studies have variously reported that the disease severity and survival in men is worse, similar, and better than in women. In contrast, there is general agreement about the influence of the age of onset of disease on survival. Most investigators have found that onset of SLE in the paediatric age group is associated with a worse outcome than in adults. Onset in the over 60 year old age group has been associated with milder disease and better survival.

The influence of race has been well documented in the United States where blacks have a consistently worse outcome than whites. It has been suggested, however, that the difference between black and white patients is more due to socioeconomic factors than to the racial characteristics.

It is to be expected that specific disease features which result in impaired organ function will influence outcome and survival. Nephritis occurs in 50 to $90 \%$ of patients with SLE depending on the criteria used to define renal disease. In a multicentre study of outcome designed to identify causes of death in 222 patients nephritis $(18 \%)$ was the most frequently observed primary cause of death resulting from active disease. ${ }^{7}$ In all, $31 \%$ of deaths were attributed to active organ involvement and $33 \%$ to infection. The central nervous system was the second most frequently involved organ where active disease was the primary cause of death. The primary cause of death was either unrelated to SLE or unknown in $22 \%$.

Measurement of survival in SLE depends on accurately identifying the time of disease onset. Many reported studies have been retrospective, depending on patients' memories and review of old case notes. The time of onset has been variously taken as the date of onset of the first symptom attributable to SLE, the development of four American Rheumatism Association criteria, the time of diagnosis, or the time of entry to the study. In this issue of the Annals Swaak and colleagues from the Netherlands have prospectively studied outcome and survival in SLE in 110 patients attending their clinics since $1970 .^{8}$ Onset of disease was defined as the time of onset of the first symptom attributable to SLE. The study evolved from the establishment of a department at the central laboratory of the Red Cross Blood Transfusion Service in Amsterdam which focused on methods for detecting antinuclear antibodies under the direction of $\mathrm{Dr}$ T E W Feltkamp. The department was associated with three collaborating institutions where special SLE clinics were developed. Prospective study protocols were designed with emphasis on assessing the significance of antibodies to double stranded DNA. In the study of Swaak $e t a l^{8}$ the clinical data relating to the disease course were extracted. The authors were particularly interested in studying differences between patients who died and those who survived. In all, 14 patients $(13 \%)$ died during the study (five male, nine female), giving a cumulative 10 year survival of $87 \%$. When survival in men was calculated the figure of $69 \%$ was considerably 
worse than the $90 \%$ calculated for women. The small numbers involved in these calculations diminish their true significance, however. Another observation in relation to survival was that the mean duration between disease onset and diagnosis was $10 \cdot 1$ years in those who died compared with 4.6 years in those alive at the end of the study. Of the causes contributing to death, nephritis was the most frequently encountered, seen in six patients $(43 \%)$. Sepsis was a cause of death in only two patients and neurological disease contributed in only one. When associations with poor outcome were sought the presence of proteinuria, cellular casts on urine microscopy, seizures, or psychosis, either at disease onset or at diagnosis, were more frequently seen in those who died. Another aspect examined in some detail was the possible association between the number of disease exacerbations and poor outcome. Thus patients who did not undergo a further disease exacerbation after the initial diagnosis had a $100 \%$ 10 year survival, whereas patients undergoing one, two, or three further exacerbations had a $91 \%$, $69 \%$, and $33 \%$ survival respectively.

As survival in SLE continues to increase more attention must be given to morbidity and the quality of life as a measure of disease outcome. Major morbidity may result from single strategic disease events such as stroke, protracted involvement of a major organ such as glomerulonephritis, or recurring manifestations such as photosensitive exacerbations, which can have important effects on lifestyle. In addition, morbidity may result from the complications of treatment. This issue of the Annals also contains two papers which address aspects of morbidity in SLE. ${ }^{90}$ A second study from the Dutch workers examined the occurrence of exacerbations during the disease course. Forty four per cent of patients had no further exacerbations after diagnosis of SLE and subsequent treatment; a further $37 \%$ had only one subsequent exacerbation. The expected range of clinical manifestations emerged during disease exacerbations, but certain trends were observed. Firstly, disease exacerbations occurred early in the course of follow up and much less frequently after five years. Secondly, disease exacerbations were considerably more likely in patients who presented with proteinuria, cellular casts, seizures, psychosis, or haemolytic anaemia at the time of diagnosis. Of the laboratory variables studied, the presence of anti-Sm antibodies or a false positive serological test for syphilis at diagnosis were indicators of likely further exacerbations. The presence of anti-DNA antibodies at diagnosis was not associated with a tendency towards further exacerbations.

The study by Wysenbeek et al examined photo- sensitivity in SLE and reported on its prevalence $\frac{\text { ? }}{\text { s. }}$ expression, and effects on lifestyle in patient attending either Stanford University or Johns? Hopkins University. ${ }^{10}$ Published reports on the incidence of photosensitivity in SLE vary consider음 ably, but photosensitivity appears to be mos? prevalent in white women and least prevalent in men of darker complexion. ${ }^{11}$ In the study of Wysenbeek et al a cohort of 125 consecutive patients with SLE were assessed and compared with $28 \mathrm{P}$ patients with rheumatoid arthritis. ${ }^{10}$ Seventy three per cent of the patients with SLE reported photos sensitive skin eruptions compared with $23 \%$ of the control subjects. Photosensitivity in SLE involveof the face predominantly $(59 \%)$ and could precede $\infty$ occur contemporaneously with, or follow onset of disease. Dubois and Wallace found that photot sensitivity was very infrequent in individuals of darlo complexion $(6.4 \%) .{ }^{11}$ Sun exposure led to an exacerbation of systemic features in $77 \%$. knowledge of the potential effects of sunlight cause $\bar{\phi}$ most patients to take protective measures, such as. avoiding sunbathing (83\%), using a strong suno screen $(70 \%)$, and avoiding direct sunlight $(68 \%)$.

Despite some encouraging trends in relation to survival SLE remains a major challenge to the skils and experience of rheumatologists. Most patient continue to depend on long term corticosteroids: immunosuppressive drugs, antihypertensive treat $\frac{\mathscr{Q}}{\square}$ ment and, increasingly, dialysis and transplantation $\overrightarrow{\vec{R}}$ Despite the information now accumulated optima $\$$ therapeutic strategies are not agreed, even in relation to nephritis, which remains the predominant. cause of death universally. Studies from individuas institutions are unlikely to be productive in advancing our knowledge on how to increase survival and improve the quality of life in SLE. More than ever there is a need for collaborative multicentre long term prospective studies using reliable measures of overall and selected organ disease activity, severity음 and response to treatment. Rheumatologists in North America have progressed some way along this route. We Europeans must now follow.

Department of Rheumatology,

St Vincent's Hospital,

Elm Park,

Dublin 4

\section{References} BARRY BRESNIHANN

1 Merrill M, Shulman L E. Determination of prognosis in chronie disease, illustrated by systemic lupus erythematosus. J Chroni Dis 1955; 1: 12-32.

2 Wallace D J, Podell T, Weiner J, Klinenberg J R, Forouzesh S尺 Dubois E L. Systemic lupus erythematosus - survival patterns experience with 609 patients. JAMA 1981; 245: 934-8. 
3 Ginzler E M, Diamond H S, Weiner M, et al. A multicentre study of outcome in systemic lupus erythematosus. J. Entry variables as predictors of prognosis. Arthritis Rheum 1982; 25: 601-11.

4 Rubin L A, Urowitz M B, Gladman D D. Mortality in systemic lupus erythematosus: the bimodal pattern revisited. $Q J$ Med 1985; 55: 87-98.

5 Hashimoto H, Tsuda H, Hirano T, Takasaki Y, Matsumoto T, Hirose S. Differences in clinical and immunological findings of systemic lupus erythematosus related to age. J Rheumatol 1987; 14: 497-501.

6 Ginzler E M, Schorn K. Outcome and prognosis in systemic lupus erythematosus. Rheumatic Diseases Clinics of North America 1988; 14: 67-78.

7 Rosner S, Ginzler E M, Diamond H S, et al. A multicentre study of outcome in systemic lupus erythematosus. 11 causes of death. Arthritis Rheum 1982; 25: 612-7.

8 Swaak A J G, Nossent J C, Bronsveld W, et al. Systemic lupus erythematosus. I. Outcome and survival: Dutch experience with 110 patients studied prospectively. Ann Rheum Dis 1989; 48: 447-54.

9 Swaak A J G, Nossent J C, Bronsveld W, et al. Systemic lupus erythematosus. II. Observations on the occurrence of exacerbations in the disease course: Dutch experience with 110 patients studied prospectively. Ann Rheum Dis 1989; 48: 455-60.

10 Wysenbeek A J, Block D A, Fries J F. Prevalence and expression of photosensitivity in systemic lupus erythematosus. Ann Rheum Dis 1989; 48: 461-3.

11 Dubois E L, Wallace D J. Clinical and laboratory manifestations of systemic lupus erythematosus. In: Wallace D J, Dubois E L, eds. Lupus erythematosus. Philadelphia: Lea and Febiger, 1987: $317-449$. 